\title{
Truss Assembly and Welding by Intelligent Precision Jigging Robots
}

\author{
Erik Komendera \\ Department of Computer Science \\ University of Colorado at Boulder \\ Boulder, CO 80309
}

Email: erik.komendera@colorado.edu

\author{
John T. Dorsey, William R. Doggett \\ NASA Langley Research Center \\ Hampton, VA 23681 \\ Email: \{john.t.dorsey,bill.doggett\}@ nasa.gov
}

\author{
Nikolaus Correll \\ Department of Computer Science \\ University of Colorado at Boulder \\ Boulder, CO 80309
}

Email: nikolaus.correll@colorado.edu

\begin{abstract}
This paper describes an Intelligent Precision Jigging Robot (IPJR) prototype that enables the precise alignment and welding of titanium space telescope optical benches. The IPJR, equipped with $\mu m$ accuracy sensors and actuators, worked in tandem with a lower precision remote controlled manipulator. The combined system assembled and welded a $2 \mathrm{~m}$ truss from stock titanium components. The calibration of the IPJR, and the difference between the predicted and the truss dimensions as-built, identified additional sources of error that should be addressed in the next generation of IPJRs in 2D and 3D.
\end{abstract}

\section{INTRODUCTION}

A hardware prototype of an Intelligent Precision Jigging Robot (IPJR), used as an aid to assemble high-precision trusses from low precision components, is presented (Figure 1). A jig is the scaffolding used to hold components in place while the welding is performed. Intelligent Precision Jigging Robots (IPJR), introduced and described extensively in [1], [2], hold multiple components in a precise pose for welding, enabling an external, imprecise agent to manipulate, assemble, and weld components made of stock materials. IPJRs are autonomous, with a comptuer containing the assembly sequence, a pose controller, algorithms for adjusting for errors, and communication hardware. An IPJR's size is not bound to structure size, so it can be reused. Trusses are typically made of repeating triangular and tetrahedral cells; therefore truss IPJRs are designed to build one cell at a time. The IPJR prototype in this paper sequentially assembles cells.

The initial prototype [1], [2] enabled the assembly of flat wooden trusses to a precision of under $1 \mathrm{~mm}$, as measured on the strut lengths, despite using simple, imprecise tools such as glue guns and hot glue. This work demonstrated that IPJRs and a coarse manipulator could work together to assemble structures made of simple elements to a high precision, thereby negating the need for the high cost associated with high precision manufacturing. The prototype presented in this paper addresses the requirements of space telescope optical benches. This prototype has actuators to grip both the nodes and the struts, enabling tele-operation of the entire process. High precision sensors and actuators are used to try to meet the precision requirement for telescope trusses, which are on the order of $\mu \mathrm{m}$. Struts and nodes are made of titanium. The external manipulator is the Lightweight Surface Manipulation System (LSMS) [3], which is tele-operated in the experiment described in this paper, and is used to weld the struts to the nodes, and maneuver the IPJR triangle.

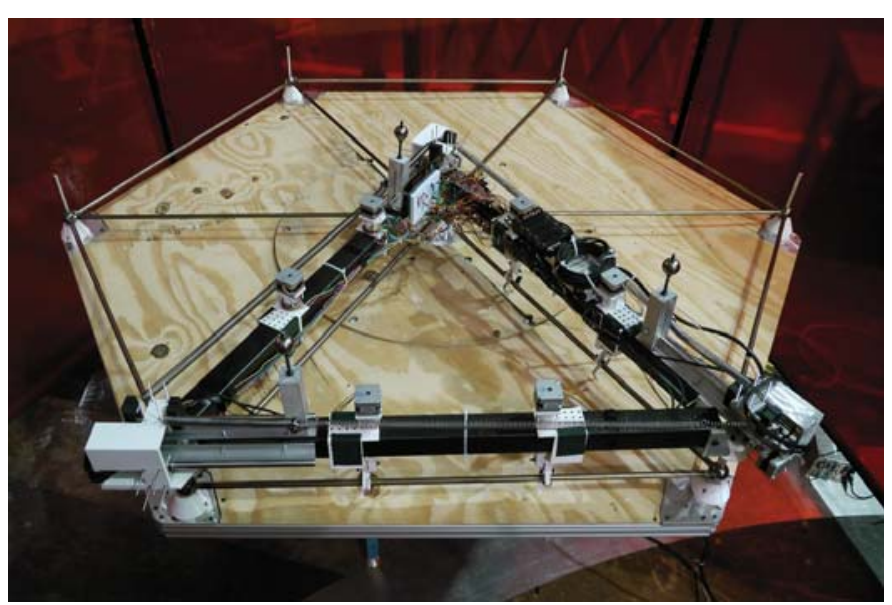

Fig. 1. The IPJR prototype is shown resting on the finished titanium truss on which the nominal distance between the tops of the node posts is 1.002 $m$.

Assembling structures in space has the potential to overcome payload limitations imposed by single-launch missions, thereby enabling the manufacturing of large and scalable structures. In particular, space telescopes that could be assembled on orbit are a high priority for NASA [4], with proposed diameters from tens of meters up to hundreds of meters. In contrast, the James Webb Space Telescope, at $6.5 \mathrm{~m}$, may be the largest telescope that can be launched preassembled. Telescope mirrors usually consist of multiple mirror elements that are mounted onto a truss that forms a parabolic surface. The truss needs to be constructed such that the mounting points for the mirrors precisely follow the desired parabolic curve. How these mounting points are connected, however, is less important, motivating the approach presented in this paper. In previous work [5], the repeated assembly and disassembly of an $8 \mathrm{~m}$ telescope mirror using an industrial manipulator arm and a rotating assembly platform was demonstrated. While this approach has worked in a laboratory environment on the ground, it is considered impractical as it requires considerable launch mass, high precision for every structural element, and is inflexible to unexpected environmental effects.

More recently, distributed field assembly by heterogenous robots has been explored in large-scale assembly tasks. Three different robots are shown to successfully dock a part to an assembly [6]. An experiment performed at JPL demonstrated 


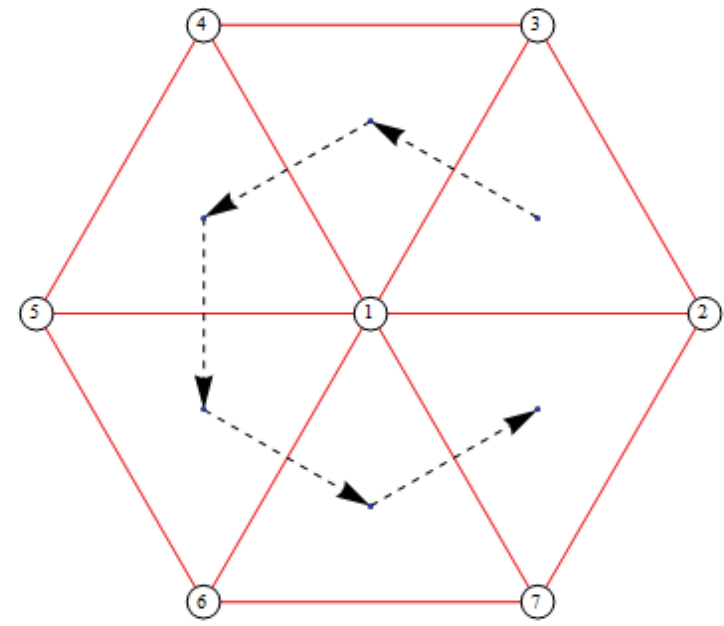

Fig. 2. The IPJR begins assembly on the upper right cell, called the kernel, and continues assembly counterclockwise. Node labels are shown; edge labels are 2-digit strings of the type $a b$ where $a$ and $b$ are the node labels, and $a<b$.

the precision assembly of beams by a pair of cooperative robots using highly rigid motions to ensure precision [7]-[9]. A robust algorithm is described in [10], which uses teams of robots to assemble structures while handling exceptions due to a wide range of failures, and relying only on a human operator when failures are beyond the scope of the assembly robots.

\section{EXPERIMENTAL SETUP}

The purpose of the experiment presented in the paper was twofold: first, to show that a stock titanium truss can be assembled and welded by an IPJR and the LSMS external manipulator, and second, to better understand the limiting factors in achieving $\mu \mathrm{m}$ precision. Although the hardware chosen has the capability to match that level of precision if all sources of error are accounted for, the experiment identified a series of design challenges that prevented this prototype from reaching the theoretical limits of sensor and actuator precision.

The IPJR truss assembly capability is demonstrated here by constructing a 2D truss made of 7 nodes and 12 struts, arranged in a hexagonal lattice of six cells, representing a simplified optical bench for a space telescope (Figure 2). The cells were nominally equilateral triangles with a node-to-node distance of $1.002 \mathrm{~m}$.

Starting with a prebuilt cell called the "kernel", which is used both as calibration target as well as a reference for the IPJR to start, assembly proceeded in five stages, repeated once for each additional cell:

- $\quad$ The LSMS lifted the IPJR off the truss and positioned it over a canister containing the new struts and nodes

- $\quad$ The IPJR captured the required struts and nodes from the canister

- $\quad$ The LSMS positioned the IPJR on the assembly site
- The IPJR adjusted its lengths to the desired truss dimensions, positioning the new node appropriately

- The LSMS welded the new struts and node to the truss

To accomplish these steps, the IPJRs required the capability to capture the truss components, the LSMS required a welding end effector and a lifting mechanism, and the canister needed to provide the new components in such a way that the IPJR could capture them. Additionally, the IPJR needed the ability to compensate for the discrepancy between the kernel and the nominal $1.002 \mathrm{~m}$ edge lengths, thus necessitating actuators to change the IPJR edge lengths. Figure 3 shows the tools used to complete the experiment.

The materials for the truss and the IPJR were chosen with attention to mitigating precision errors. Titanium was chosen for its favorable low coefficient of thermal expansion of 8.5 $\frac{\mu m}{m^{\circ} \mathrm{C}}$, an important consideration for telescopes on orbit subject to thermal expansion. Struts were $12.7 \mathrm{~mm}$-diameter hollow tubes of $961.9 \mathrm{~mm}$ length, which were welded to node balls $38.1 \mathrm{~mm}$ in diameter. Affixed to the top of each node ball was a $12.7 \mathrm{~mm}$-diameter, $107.95 \mathrm{~mm}$-tall aluminum post. Each post represented a mounting point for a segment of the reflector. The node post positions were the metric for determining the precision of the truss.

The Lightweight Surface Manipulation System (LSMS) is a long-reach manipulator designed to operate on planetary surfaces, intended to manipulate large objects in preparation for a manned landing [3]. It is $4.25 \mathrm{~m}$ tall and has a reach of $8.5 \mathrm{~m}$. By itself, the LSMS provides precision on the order of $\mathrm{mm}$, which is not sufficient to construct trusses at the precision demanded by space telescopes. An off-the-shelf arc welding gun was integrated into an end effector attached to the LSMS wrist. A lifting plate end effector gave the LSMS the ability to lift the IPJR by grasping three attachment points on the IPJR.

The limited maneuverability of the LSMS requires a turntable to provide the final degree of freedom necessary for controlled placement of the IPJR and the welding gun. In this experiment, a human operator rotated the turntable as necessary, but future experiments will utilize a motorized turntable that will be under control of the IPJR.

The canister presented new struts and nodes for the IPJR to capture while the LSMS positioned the IPJR over the canister. It was built and positioned in such a way that the struts and nodes are positioned roughly in the shape of the cell they are supposed to build. This enabled the IPJR to grab all of the required elements at once and carry them over to the assembly site. As with the turntable, this version required a human operator, but future versions will utilize motors controlled remotely by the IPJR.

\section{A. Intelligent Precision Jigging Robot}

The Intelligent Precision Jigging Robot in this paper was nominally designed (Figure 4) to align the tops of the node posts (where a mirror would be attached) to within $5 \mu \mathrm{m}$ of an adjustable length in the range of 0.987 to 1.013 meters. To meet the $5 \mu \mathrm{m}$ requirement, the IPJR uses Ultra Motion D-Series actuators, with a specified $7.9 \mu \mathrm{m}$ motion per step, enabling any desired length to be within $3.95 \mu \mathrm{m}$ of a step, and a $50.8 \mathrm{~mm}$ stroke length. To test repeatability and to calibrate, 

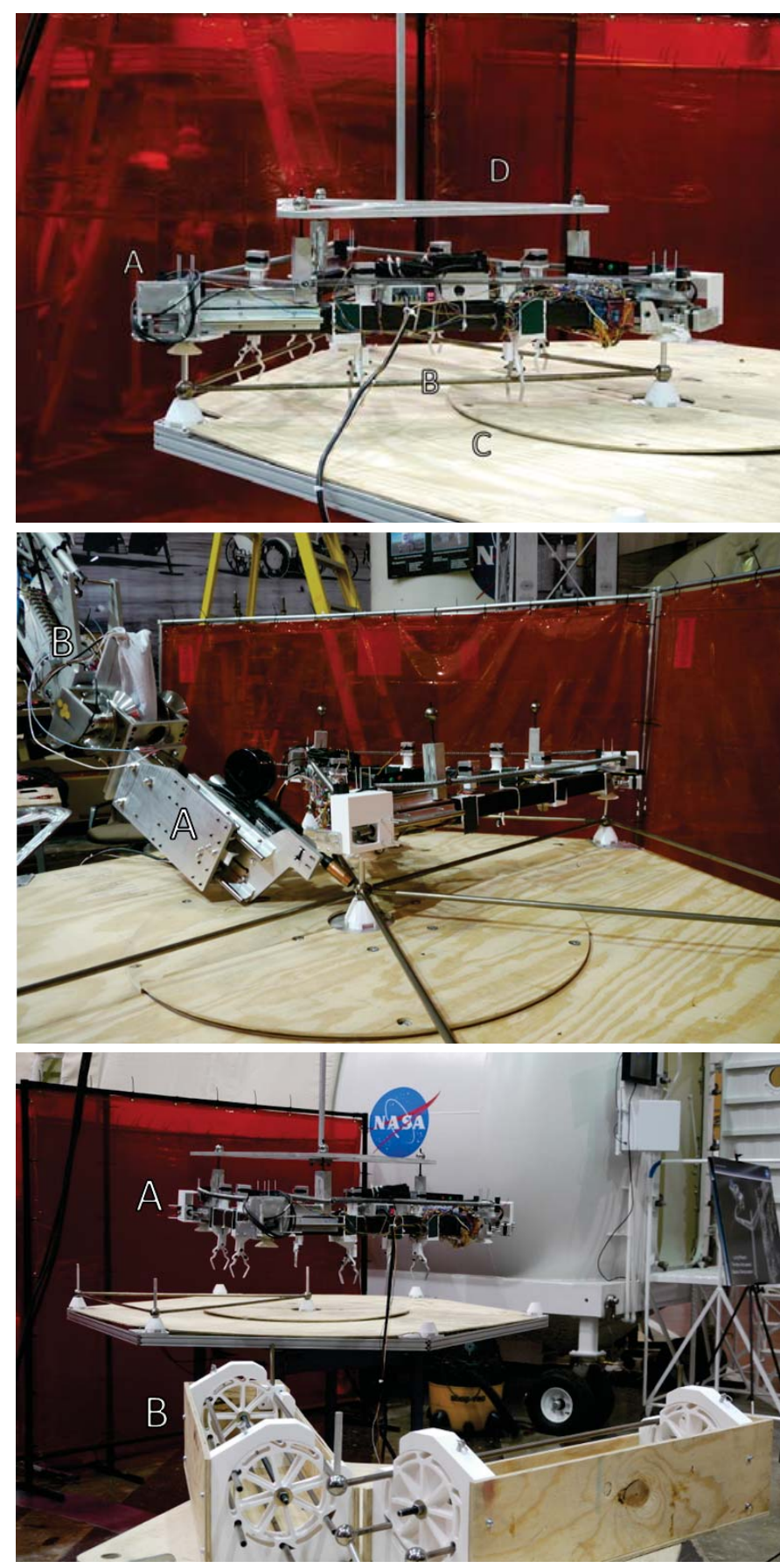

Fig. 3. Top: The IPJR (A) rests on top of the kernel (B) at the start of the experiment, which in turn rests on the turntable $(\mathrm{C})$; the lifting plate (D) is used to lift the IPJR. Middle: The welding end effector (A), attached to the LSMS forearm (B), is preparing to weld strut 16 to node 1. Bottom: The LSMS is transporting the IPJR (A) to the strut canister (B) in the foreground.

each edge had a Keyence IL-030 laser distance sensor to sense the length of the IPJR edge by measuring the extension of the Ultra Motion actuator. The IL-030 has a repeatability of $1 \mu \mathrm{m}$, but a small operational range of $20-45 \mathrm{~mm}$, which limited the range of the IPJR.

The IPJR is a triangle consisting of three identical edges, with the exception of auxiliary hardware attached to one of

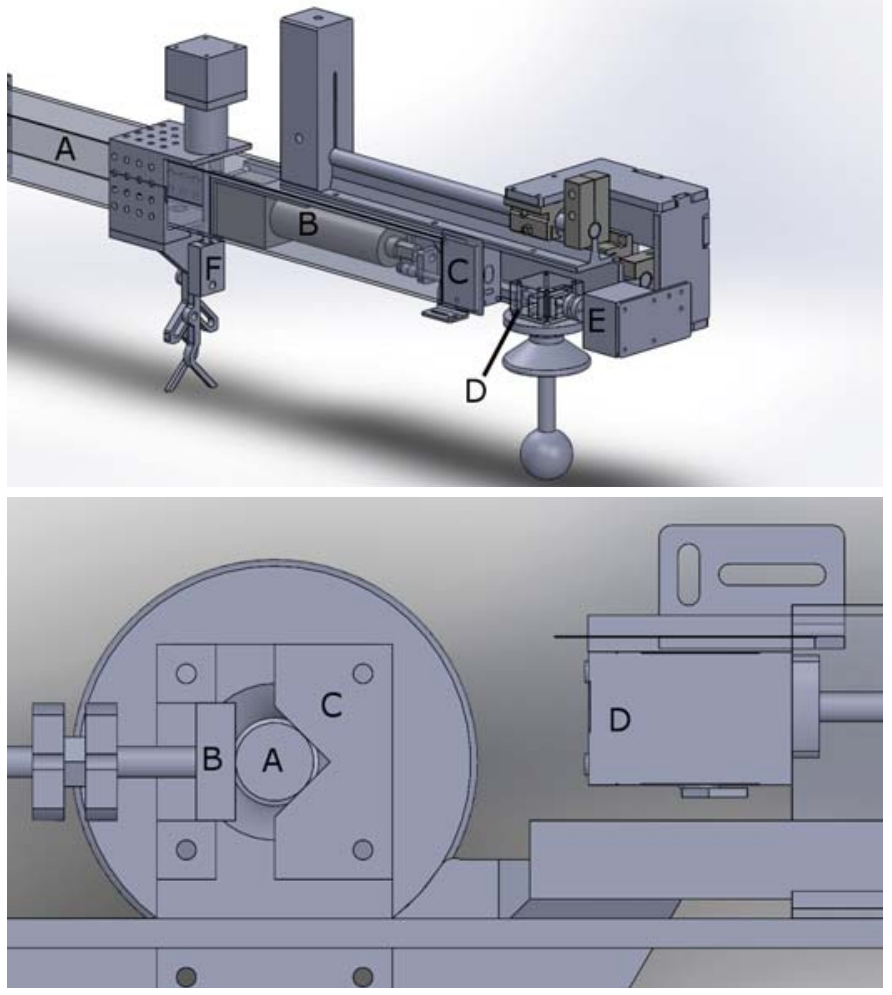

Fig. 4. Top: The composite tube (A) contains the Ultra Motion actuator (B) and the IL-030 (C); the IL-030 measures its distance from the target plate (D), which also captures node posts using a stepper motor $(\mathrm{E})$; another gripper $(\mathrm{F})$ captures struts. Bottom: A top-view cutaway shows the node post (A) being pushed by the stepper motor (B) into a right-angled surface on the target plate (C), which is also used for distance sensing by the IL-030 (D).

the three edges. Each edge is a mechanical linkage between two components, the main body and the node gripper module. The main body of each edge is a composite tube, a material chosen for its favorable thermal expansion properties over most metals. An Ultra Motion actuator extends the node gripping module away from the main body. Two rails with bearings are used to prevent bending moments on the Ultra Motion actuators, which can cause them to fail under lateral loads of just $13 \mathrm{~N}$.

The node gripping module consists of a funnel used for guiding a node post while the IPJR is being lowered onto the canister, a target plate for capturing node posts precisely, and a stepper motor to push the node post into the target plate. The IL-030, attached to the main body, measures the edge's extension by measuring its distance to the target plate. Attached to the tube are two grippers for lifting and holding the struts.

To eliminate free play within the entire IPJR, springs are attached between the lifting plate posts, imposing a compressive force on each edge. Thus, when each Ultra Motion is commanded to go to a step, there will be no loose components to interfere with the measurement.

\section{CALIBRATION}

Prior to the experiment, the kernel was measured on a quality assurance instrument at NASA, accurate up to $0.1 \mu \mathrm{m}$. 


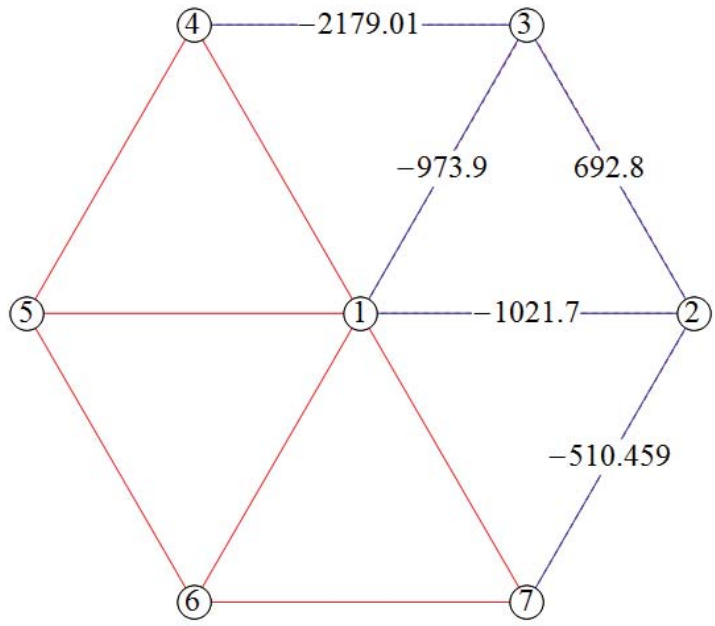

Fig. 5. The kernel node post position errors required the IPJR to adjust it lengths on the blue edges from the nominal $1.002 \mathrm{~m}$ by the value (in $\mu \mathrm{m}$ ) shown. Unaffected struts are shown in red.

The nominal kernel edge lengths were $1.002 \mathrm{~m}$. The distances between the kernel posts were found to be $1021.7 \mu \mathrm{m}$ too short on edge $12,692.8 \mu \mathrm{m}$ too long on edge 23 , and $973.9 \mu \mathrm{m}$ too short on edge 13. To restore the remaining nodes to their correct positions, the IPJR length setting for edges 34 and 27 had to be adjusted by 2179 and $-510 \mu m$ respectively. Figure 5 shows the modified truss settings based on the kernel.

To calibrate the IPJR on the kernel, touch sensors were applied to the node posts on the kernel and the target plates. Each edge length had some influence on whether or not contact was made on the others, so the edges could not be calibrated one at a time. This amounted to a 3-dimensional search for a maximum contact-free length for all three actuators such that the slightest increment in one edge would result in contact on that edge only. For each edge, that step count is saved as $s_{t e p_{e, c a l}}$ for the kernel edge length $L_{e, c a l}$ plus the thickness of the touch sensors, $0.6 \mathrm{~mm}$. The IL-030 distance sensor was used to measure the extension of the edges during the calibration, resulting in an expected distance-per-step for each actuator, or $r_{e, c a l}$. Once these values are known, the actuator extension function was defined as:

$$
\operatorname{step}_{e}(L)=\frac{L-L_{e, c a l}}{r_{e, c a l}}+\operatorname{step}_{e, c a l}
$$

The individual edges of the IPJR are labeled IPJR 1, 2, and 3. IPJR 1 was positioned over edge 13, IPJR 2 over edge 12, and IPJR 3 over edge 23. Respectively, step $p_{e, c a l}$ was 160, 310, and 425 steps. The calculation of $r_{e, c a l}$ involved finding a least squares fit on the laser measurements, with results shown in Figure 6. However, at shorter lengths, as the IPJR was closing in on the maximum contact-free lengths, the mutual effect each edge had on the other resulted in significant nonlinearity (the green points). To get a good estimate for $r_{e, c a l}$, the slope was only calculated for lengths sufficiently distant from the

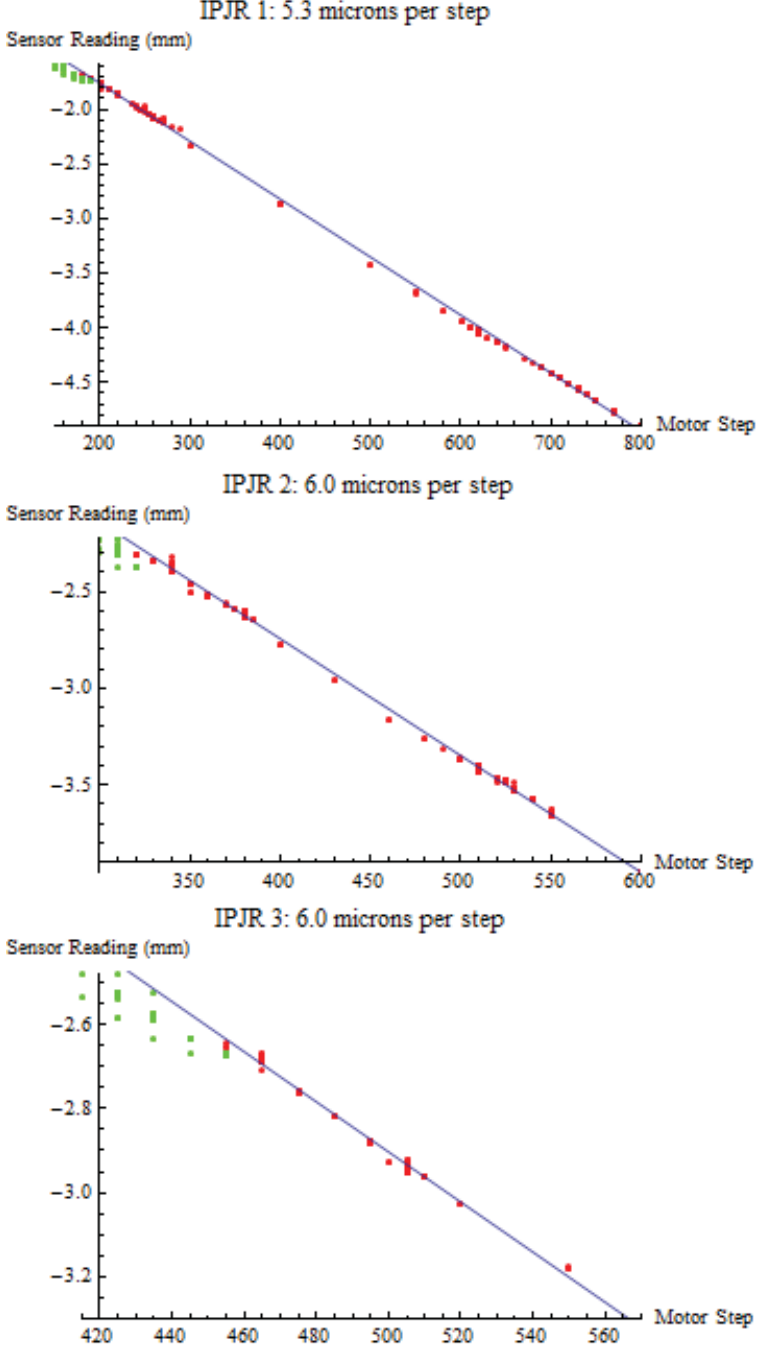

Fig. 6. Results of the laser calibration, in which the steps from full retraction were mapped to the sensor readings. The sensors were arbitrarily zero-shifted, but only the relevant readings were of interest. The nonlinear region is shown with green points and the quasi-linear region with red points. The distanceper-step value for each IPJR edge, $r_{e, c a l}$, was calculated using the red points.

nonlinear regions (the red points). The result is a much tighter fit on the red points. $r_{e, c a l}$ was found to be 5.3,6.0, and $6.0 \mu \mathrm{m}$ respectively. Note that these values differ from the specified Ultra Motion step distance of $7.9 \mu \mathrm{m}$. Reasons may include the compressive force from the springs and the node posts when the IPJRs are overextended, thereby contracting the parts of each edge, and the IL-030s being slightly off-axis.

The nonlinear data points were included in the calculation of the standard deviation $\sigma_{e, s}$. For IPJR 1, 2, and 3, the standard deviations were $35 \mu \mathrm{m}, 40 \mu \mathrm{m}$, and $34 \mu \mathrm{m}$. When the nonlinear points are excluded, these become $26 \mu \mathrm{m}, 18$ $\mu m$, and $11 \mu m$, showing that this prototype cannot meet the $5 \mu \mathrm{m}$ precision goal, thus necessitating changes in the next prototype.

\section{Probability Model and Error Prediction}

The physical model was simplified by considering all IPJR edges to be identical, assuming zero mean error and multi- 
variate Gaussian distribution on both the IPJR edge lengths and the physical errors arising from the welding process. The joint probability distribution function was composed of two kinds of conditional probability distributions: the probability distribution of the edge length settings given the desired length, and the probability distribution of the actual node position given the edge length settings. Formally, the IPJR edge length variable, $s_{i k}$, is defined as:

$$
p\left(s_{\mathrm{ik}}\right)=\mathcal{N}\left(L_{\mathrm{ik}}, \sigma_{s}^{2}\right)
$$

Where $L_{\mathrm{ik}}$ is the desired length of edge $i j$ (nominally 1.002 $m$, modified as shown in Figure 5), and $\sigma_{s}$ is the maximum standard devation found by calibration to be $40 \mu \mathrm{m}$. The mean of the position of the next node, $\hat{x_{k}}$, depends on $s_{\mathrm{ik}}, s_{\mathrm{jk}}, \overline{x_{i}}, \overline{x_{j}}$. It is too long for print, but can be trivially found by applying the law of cosines to the triangle formed by the inputs. The variance of $\overline{x_{k}}$ assumes that error is equally possible on both $x$ and $y$ dimensions (in addition to the strut-vector-specific variances of the edge lengths). The probability is therefore:

$$
p\left(\overline{x_{k}} \mid s_{\mathrm{ik}}, s_{\mathrm{jk}}, \overline{x_{i}}, \overline{x_{j}}\right)=\mathcal{N}\left(\hat{x_{k}},\left(\begin{array}{cc}
\sigma_{n}^{2} & 0 \\
0 & \sigma_{n}^{2}
\end{array}\right)\right)
$$

The joint probability distribution function is the product of all of the conditional distributions and is shown in probabilistic graphical model form in Figure 7. The computational complexity of the full probability distribution function made it necessary to estimate the marginal distributions of the node positions using Monte Carlo trials. To predict a theoretical best possible 95th percentile ball centered on the desired node positions given the calibration variance, the assembly was sampled 5000 times with $\sigma_{s}=40 \mu \mathrm{m}, \sigma_{n}=0 \mu \mathrm{m}$. The 95th percentiles of each node are shown in Figure 8. Assuming everything else is perfect, the calibration shows that the best that this IPJR prototype could accomplish is to set the final node within $248 \mu \mathrm{m}$ of the desired point with $95 \%$ certainty, far from the long term goal, indicating that a more predictable and reliable design is needed for the next prototype.

\section{EXPERIMENT}

The assembly of the truss took place over six hours, with the full truss being completed by the IPJR and the LSMS. The maneuvering and welding by the LSMS contributed most of the duration. To save time, the strut canister was not used for the final 4 cells, since it was shown to work at least once and otherwise did not affect the outcome of the experiment. Upon completion, the experimental setup was disassembled and the truss was measured. The measurements are shown in Table I and Figure 9.

The data show that, compared to the desired $1.002 \mathrm{~m}$ separation, all of the nodes are less than $1 \mathrm{~m}$ apart. The largest deviation is on node 3 , on the kernel, at $5.03 \mathrm{~mm}$, and among the nodes placed by the IPJR, the largest deviation is on node 4 at $4.55 \mathrm{~mm}$. The edge lengths show a range of $2.207 \mathrm{~mm}$, with a standard deviation of $593 \mu \mathrm{m}$.

The data not only significantly differs from the desired structure, but the kernel substructure differs as well, and the

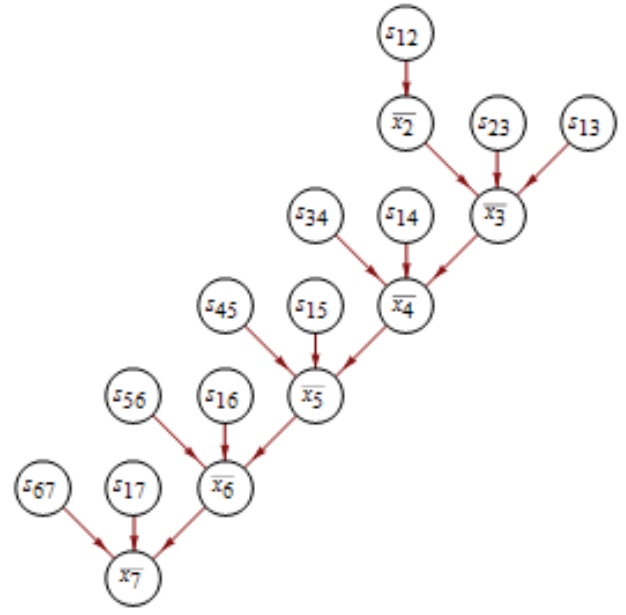

Fig. 7. Probabilistic graphical model of the node positions in which random variables depend conditionally on the random variables pointing to it. $\overline{x_{1}}$ is the origin and not a random variable, and so is omitted.

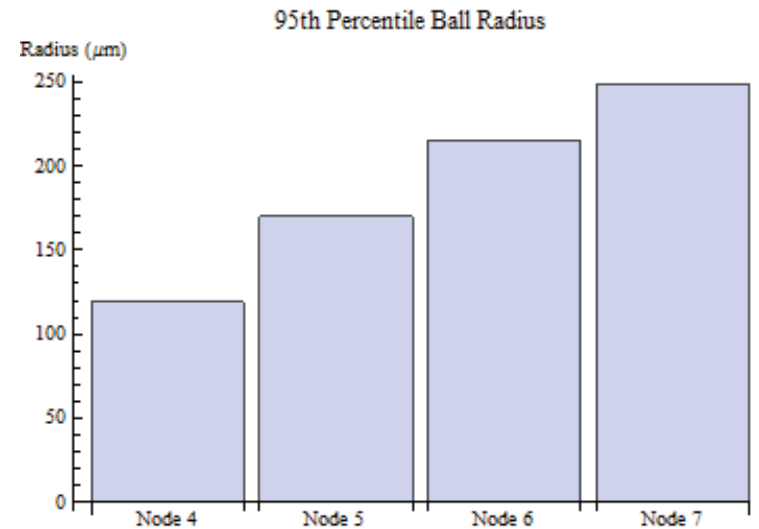

Fig. 8. Five thousand Monte Carlo trials of the Bayesian network model of the assembly process, with $\sigma_{s}=40 \mu \mathrm{m}$ and an ideal node placement error $\sigma_{n}=0$, show that the 95th quantile is at worst $248 \mu \mathrm{m}$ from the desired point.

relationship is not simply a factor of scale. Strut bowing was observed, indicating tensions and compressions within the truss, possibly arising from post-weld thermal contraction.

\section{Discussion AND Future WORK}

A system consisting of an IPJR and the LSMS successfully assembled and welded a truss using stock titanium materials, and improving on the methods and practicality of the wooden experiment from [1], [2]. The LSMS was augmented with a welding gun and a lifting plate for the experiment. The IPJR successfully retrieved the next cell's struts and nodes from a canister. The LSMS lifted and placed the IPJR properly, resulting in a correct capture of the node posts on the truss.

Although the experiment was a successful demonstration of an IPJR working with the LSMS to assemble and weld a truss structure, the approach needs modification to meet the 
TABLE I. NODE COORDINATES AND DISTANCE ERROR AFTER $\operatorname{ASSEMBLY}(m)$

\begin{tabular}{|l|l|l|l|}
\hline $\mathrm{x}$ & $\mathrm{y}$ & $\mathrm{z}$ & $\|$ error $\|$ \\
\hline 0. & 0. & 0. & 0. \\
0.998072 & 0. & -0.00004572 & 0.0039282 \\
0.497226 & 0.864428 & -0.0000127 & 0.00503288 \\
-0.501045 & 0.863276 & -0.00078486 & 0.00454969 \\
-0.998114 & -0.00196461 & 0.00079756 & 0.00442672 \\
-0.498673 & -0.866141 & 0.00156972 & 0.00323913 \\
0.499244 & -0.865118 & 0.001143 & 0.00337018 \\
\hline
\end{tabular}

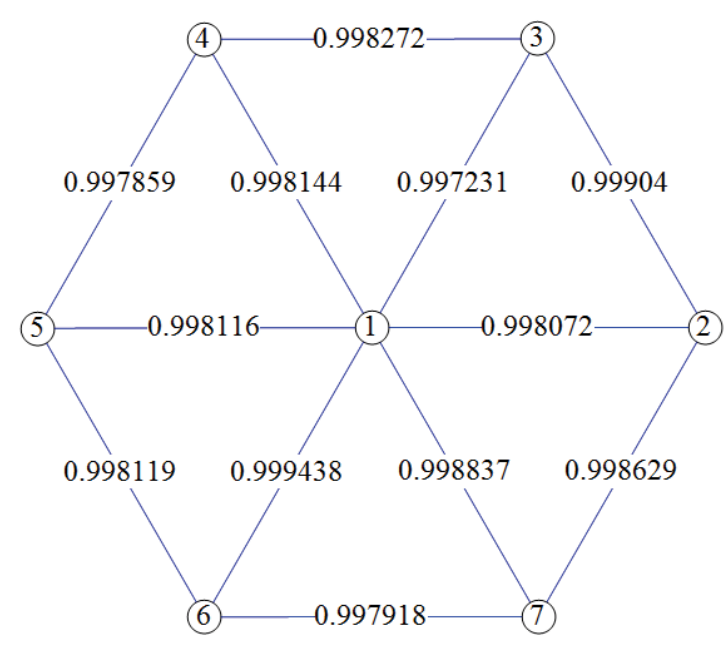

Fig. 9. The measured distances between the nodes (m) on the final truss structure.

precision requirement. As intended, the experiment revealed several issues which will lead to modifications. The use of high precision actuators is promising, but it will rely on a much more accurate measurement than the IL-030 sensors provided. The sensors measured a small gap between the main body and the node gripper body, which only gave a measurement of the length of the gap, and can only scale reliably to the IPJR as a whole assuming the structure components are rigid bodies, and the laser is perfectly aligned with the strut axis. The IPJR had a great deal of compliance, was built imperfectly, and was subject to other nonlinearities in the calibration. The calibration showed that even if the welding process introduced no errors, the 95th percentile radius from the desired node position is $248 \mu \mathrm{m}$. The measured final truss shows that all of the node distances were shorter than expected, the kernel's dimensions were altered, and bowing was visible on the struts. The maximum error of a node from its desired position is 5.03 $\mathrm{mm}$, and the lengths of the edges are all within $2.207 \mathrm{~mm}$ of one another. The processes leading to these errors could include factors such as temperature gradients (including during and after the welding process), internal forces in the structure induced by the cooling of the truss, an imprecise turntable, imprecise node posts, forces induced by the IPJRs while it was gripping the nodes, a biased calibration, and the node posts' deviation from vertical.

The next attempt at building an IPJR prototype will use a surveying system to monitor the precise distances between nodes during assembly. This enables the detection of deflections due to all of the causes listed above, in addition to gravity, which will be a factor in 3D assembly experiments. A surveying system can be used to better calibrate the next IPJR. Such a survey system may also be distributed, requiring sensor fusion to combine the distributed data into a better estimate. The size of a truss structure would not be limited by using a single surveyor.

The practicality of the IPJR paradigm to assemble truss structures, especially on orbit, will depend on how well the IPJR system can identify and correct errors that build up in the system. That said, the first trial of a welded structure made by the LSMS and the IPJR performed better than expectations, and inspires confidence that future iterations will continue this trend.

\section{ACKNOWLEDGMENTS}

This work was supported by a NASA Space Technology Mission Directorate Space Technology Research Fellowship.

\section{REFERENCES}

[1] E. Komendera, D. Reishus, J. T. Dorsey, W. R. Doggett, and N. Correll. Precise truss assembly using commodity parts and low precision welding. In Proceedings of the Fifth Annual IEEE International Conference on Technologies for Practical Robot Applications, 2013.

[2] E. Komendera, D. Reishus, J. T. Dorsey, W. R. Doggett, and N. Correll. Precise truss assembly using commodity parts and low precision welding. Journal of Intelligent Service Robotics, Accepted for Publication, 2014.

[3] J. T. Dorsey, T. C. Jones, W. R. Doggett, J. S. Brady, F. C. Berry, G. G. Ganoe, E. J. Anderson, B. D. King, and C. D. Mercer. Recent developments in the design, capabilities and autonomous operations of a lightweight surface manipulation system and test bed. In Proceedings of the AIAA Space Conference, 2011.

[4] J. J. Watson, T. J. Collins, and H. G Bush. Construction of large space structures at NASA Langley Research Center. In IEEE Aerospace Conference, 2002.

[5] W. Doggett. Robotic assembly of truss structures for space systems and future research plans. In Aerospace Conference Proceedings, 2002. IEEE, volume 7. IEEE, 2002.

[6] R. Simmons, S. Singh, D. Hershberger, J. Ramos, and T. Smith. First results in the coordination of heterogeneous robots for large-scale assembly. In Experimental Robotics VII, pages 323-332. Springer, 2001.

[7] A. Stroupe, T. Huntsberger, A. Okon, and H. Aghazarian. Precision manipulation with cooperative robots. In L. Parker, F. Schneider, and A. Schultz, editors, Multi-Robot Systems: From Swarms to Intelligent Automata, 2005.

[8] A. Stroupe, T. Huntsberger, A. Okon, H. Aghazarian, and M. Robinson. Behavior-based multi-robot collaboration for autonomous construction tasks. In Intelligent Robots and Systems, 2005.(IROS 2005). 2005 IEEE/RSJ International Conference on, pages 1495-1500. IEEE, 2005.

[9] A Stroupe, Terry Huntsberger, Brett Kennedy, Hrand Aghazarian, E Baumgartner, Anthony Ganino, Michael Garrett, Avi Okon, Matthew Robinson, and $\mathrm{J}$ Townsend. Heterogeneous robotic systems for assembly and servicing. In 8th International Symposium on Artificial Intelligence, Robotics and Automation in Space, ESA. ESA, 2005.

[10] F. W. Heger. Assembly Planning in Constrained Environments: Building Structures with Multiple Mobile Robots. PhD thesis, Carnegie Mellon University, 2010. 